\title{
A Report on the Recent Demonstration of NOAA's Upgraded Capability to Derive Cloud Motion Satellite Winds
}

\author{
Robert T. Merrill ${ }^{+}$, W. Paul Menzel*, Wayman Baker**, \\ James Lynch ${ }^{\star \star \star}$, and Eugene Legg ${ }^{\star \star \star \star}$
}

\section{Introduction}

Cloud motions apparent in a sequence of geostationary satellite images represent an important source of meteorological information, especially over the oceans. However, improvements in data assimilation and numerical weather prediction (NWP) have outpaced improvements in satellite derived cloud motion vector (CMV) production over the past decade, and the reduced impact of CMV's has caused the National Meteorological Center (NMC) to restrict and the European Centre for Medium-range Weather Forecast (ECMWF) to discontinue the use of Northern Hemisphere upper level vectors from the U.S. GOES and the Japanese Himawari. The primary reason cited is a large slow bias, especially in and near jet cores. High level vectors from the European METEOSAT are still used.

Currently, the main problem of CMV production is assigning cloud tracked motions to the correct heights. Thin clouds which are most likely to be passive tracers of the flow at a single level are the best tracers, but assigning them to the correct height is especially difficult. Since the emissivity of the cloud is less than unity by an unknown and variable amount, its brightness temperature $(\mathrm{Tb})$ in the infrared window is an overestimate of its actual temperature. Thus, heights for thin clouds inferred directly from the observed $\mathrm{Tb}$ and an available temperature profile are systematically low, and must be empirically or subjectively adjusted.

Height assignment errors carry a heavy penalty because of vertical wind shear, which is greatest near

\footnotetext{
${ }^{+}$Cooperative Institute for Meteorological Satellite Studies, Madison, Wisconsin

*NOAA/NESDIS Advanced Satellite Products Project, Madison, Wisconsin DC

**NOAA/NWS National Meteorological Center, Washington,

${ }^{\star \star \star}$ NOAA/NESDIS Synoptic Analysis Branch, Washington, DC
}

C1991 American Meteorological Society regions of active weather. For this reason, recent winds research at the Cooperative Institute for Meteorological Satellite Studies (CIMSS) has emphasized improved height assignments, and a physically based two-channel method using the " $\mathrm{CO}_{2}$ slicing" approach (Menzel et al. 1983) has been incorporated into the CIMSS experimental winds algorithm (Merrill 1989).

Following encouraging tests in February and April 1989 , a demonstration of the upgraded winds capability in a simulated operational setting was conducted in April of 1990. This report describes the upgrades and demonstration. Participating organizations are listed in Table 1.

\section{Operational winds production at NESDIS}

Low-level winds are currently produced automatically using a "picture-pair" correlation algorithm (Green et al. 1975) for the full disk from window channel imagery. Middle- and upper-level winds are produced interactively on the VAS Data Utilization Center (VDUC) computer. The operator examines three successive images at 30-min intervals and selects and tracks a feature using a joystick; then refines the feature's motion using an objective pattern matching technique (McIDAS Applications Guide 1988). A "single pixel" tracking mode is also available; the pattern match is bypassed and the tracer positions entered by the operator are converted directly to a velocity. Because of memory limitations, the full disk is processed in 10 sectors at $8-\mathrm{km}$ resolution.

Height assignments are also made interactively. The operator estimates the cloud temperature from the window channel in opaque portions of a group of clouds and determines a pressure by comparison with a collocated temperature profile (using the 6-h or 12-h forecast from the most recent aviation model run). This height is then assigned to all consistent motions associated with the cloud group. In areas of thin cloud where observed window channel temperatures are all clearly too warm, the operator assigns an 
TABLE 1. Organizations participating in the NESDIS/NWS/CIMSS winds demonstration, April 1990.

Cooperative Institute for Meteorological Satellite Studies, University of Wisconsin-Madison:

- Concept development

-Software development

- Transfer to VDUC

-Real-time demonstration winds production on VDUC

NOAA/NESDIS Interactive Products Branch:

- Demonstration software implementation on VDUC

- Software to send winds to NMC

NOAA/NESDIS Synoptic Analysis Branch:

- Demonstration winds editing on VDUC

(in addition to operational duties).

NOAA/NWS/NMC Development Division:

-Design and operation of parallel data assimilation cycle -Interpretation of model impact.

adjusted height based on the motion of the vector(s) relative to the overall synoptic situation and neighboring cloud groups with more reliable heights.

The operator also has the option to display the CMV's produced by the picture pair calculations and delete rogues. Manual quality control is implicit in the interactive winds production; the operator selects only those features with a definite motion signature and deletes any not representative vectors.

\section{The improved winds algorithm}

The long-range goal of NESDIS cloud motion winds research is to automate winds production as much as possible, freeing meteorologists to spend more time on the more complex processes and decisions. Winds processing involves tracer selection, tracking height assignment, and quality control. The current upgrade package largely automates tracer selection and height assignment. Tracking is already automated, and work continues on developing an algorithm which will perform most, but not all, of the quality control.

\section{a. $\mathrm{CO}_{2}$ ratio height assignment}

The most important upgrade to the NESDIS winds capability is a two-channel " $\mathrm{CO}_{2}$ ratio" height assignment (Menzel et al. 1983, Eyre and Menzel, 1989) using the 13.3 micron channel (VAS band 5) and the long-wave infrared window channel (VAS band 8). The details of implementation are described in Merrill (1989). The technique has been proven to be able to detect semitransparent thin clouds and to properly assign a cloud top pressure. Because of the use of a simple radiative transfer model and only two channels, difficulties still arise when multiple cloud layers are still present, especially very thin cirrus over a low-level marine cloud layer.

The algorithm also estimates a height directly from the window channel brightness temperatures from the colder portions of a tracer scene. The tracer is assigned to the higher of the two heights, but not higher than $150 \mathrm{hPa}$.

\section{b. Automated winds algorithm}

A flow chart of the automated winds algorithm is shown in Fig. 1 and its operation is summarized below. A detailed description may be found in Merrill (1989).

A target selector divides the entire image into a number of cells (100 - $200 \mathrm{~km}$ on a side), and within each, selects a point associated with a maximum in brightness temperature and gradient, subject to some conditions on the overall brightness temperature and contrast of the scene. Heights are also computed at this stage.

The tracking algorithm is then applied for each target point. A tracking area about $(100 \mathrm{~km})^{2}$ is centered on the target in the first image. The algorithm then goes to the second image in the loop and searches for the area which best matches the radiances in the tracking area. The search is confined to a $(150 \mathrm{~km})^{2}$ region centered on the displacement indicated by the 12-h NMC global aviation model wind prognosis. If a successful match is found, the indicated displacement is used to position a new tracking area on the second image and a new search area on the third, and the process is repeated. The two vectors are then averaged to produce a final wind estimate.

Three forms of quality control are applied. Because of the limited search area, motions deviating from the guess by more than a finite amount cannot be matched (Merrill 1989), weakly constraining the winds to a guess. The pair of vectors produced from the three images are compared, and if they differ by $5 \mathrm{~ms}^{-1}$ or more in either component, the report is flagged as unusable. The remaining winds are then displayed on the VDUC and edited manually by checking for consistency and by comparing with the first guess and rawinsondes (if available).

\section{Demonstration}

The goals of the April 1990 demonstration were a) to demonstrate the capability of the upgraded system to produce regional winds coverage within the operational data cut-off times, and b) to test the impact of the winds with improved heights on the NMC global forecast system. 


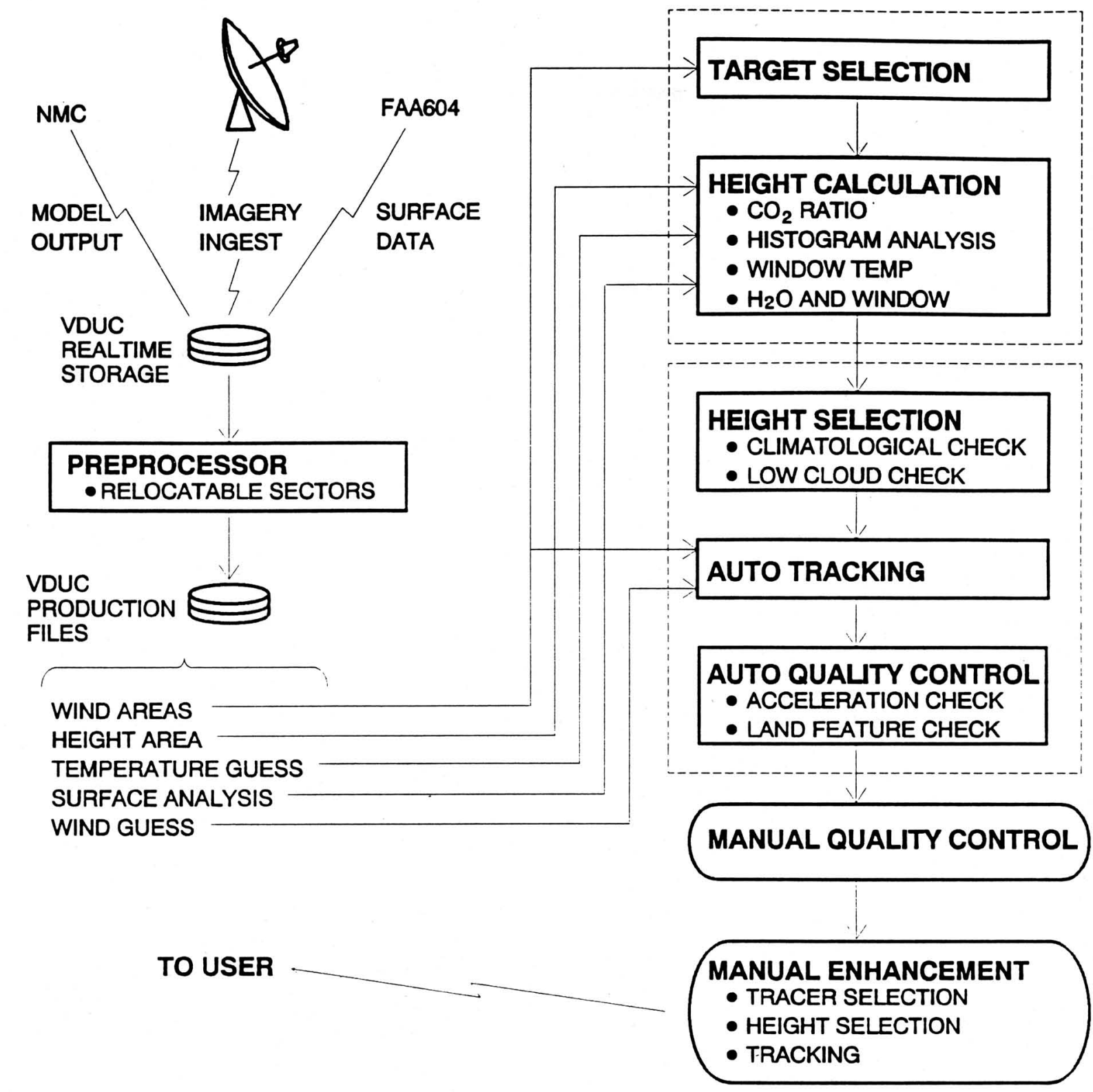

FIG. 1. Flow chart of the CIMSS experimental wind system. Boxes enclose processes, and dashed outlines define single job steps on the UW McIDAS.

The demonstration consisted of parallel production of winds using the operational and upgraded system. The domain for demonstration winds production was chosen to provide winds over the data sparse subtropical eastern Pacific Ocean in connection with the polar and subtropical jet stream and cut off lows (Fig. 2). Because the main impact of the $\mathrm{CO}_{2}$ heights is felt for high level tracers, the demonstration winds were produced only above $600 \mathrm{hPa}$. Winds were produced from 15-min loops of imagery starting at 1046, 1646, and 2246 UTC each day beginning on 18 April and continuing through 27 April. Processing was usually completed between 10 and 20 min after nominal synoptic time, and manual editing typically took $1-2 \mathrm{~h}$ beyond that.

Figures 3-5 show an example of a demonstration wind set. Figure 3 shows the operational winds. The CMV's are faster than the analysis and provide better definition of the subtropical jet along the poleward edge of the cirrus band. Figure 4 shows how the more 
accurate objective height assignments possible with the $\mathrm{CO}_{2}$ ratio method, especially in the translucent cirrus in the polar jet off of California, greatly increases the coverage possible in the demonstration wind set (Fig. 5) without subjective height reassignment.

\section{Preliminary results of the GDAS Model impact test}

The NMC Global Data Assimilation System (GDAS) was run in parallel in real time with a) the operational winds and $b$ ) the experimental winds covering the demonstration domain and operational winds elsewhere. The experimental, highlevel $(p<600$ hPA) CMV's were used in the NMC parallel GDAS at 0000,1200 , and 1800 UTC for the 10-day demonstration period. Lowlevel winds produced by the operational picture-pair technique were passed to the GDAS, even in the experimental area. Analyses produced during the assimilation were used as initial conditions for 5-day forecasts starting at 0000 UTC on April 19 through April 27.

Comparison of the parallel forecasts indicated a slight positive impact of the demonstration winds. The 5 -day $500 \mathrm{hPa}$ height forecast anomaly correlation scores for the10-day period over the northern hemisphere extratropics, a typical index of forecast accuracy, increased from 0.73 to 0.75 on average, with improvements on six of the 10 demonstration days. Though no formal significance tests are available, such a difference is equivalent to extending the limit of

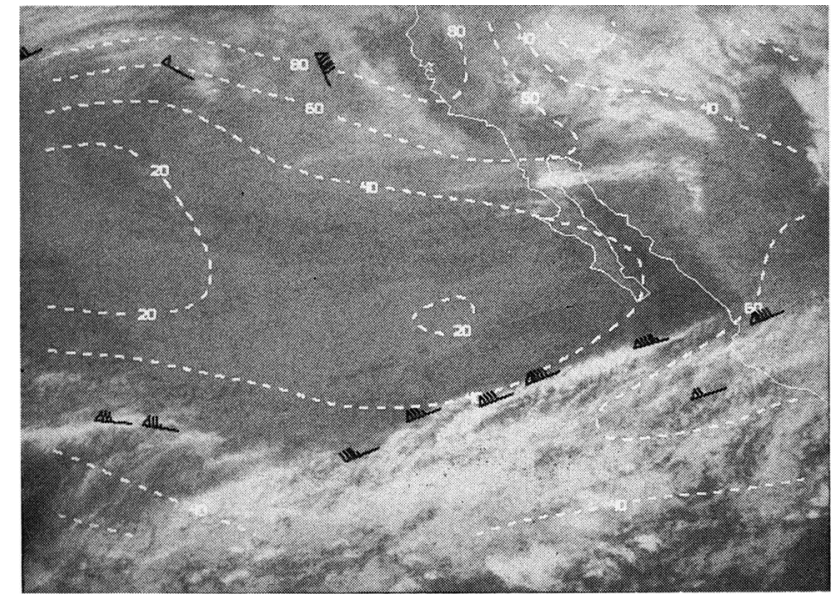

FIG. 3. Operational $200-300-m b$ winds for 0000 UTC 25 April 1990 with the NMC $250-\mathrm{mb}$ isotach analysis superimposed on the GOES infrared window image. useful forecast time by about $2 \mathrm{~h}$ and is of the same scale as difference between the forecasts made by the different global forecast centers.

\section{Operational implementation at NOAA NESDIS/SAB}

The NESDIS Synoptic Analysis Branch (SAB) used this improved winds capability for operational support of the National Hurricane Center during the 1990 hurricane season. $\mathrm{CO}_{2}$ automated winds, automated water vapor motion winds ( 6.7 and 7.3 micron bands),

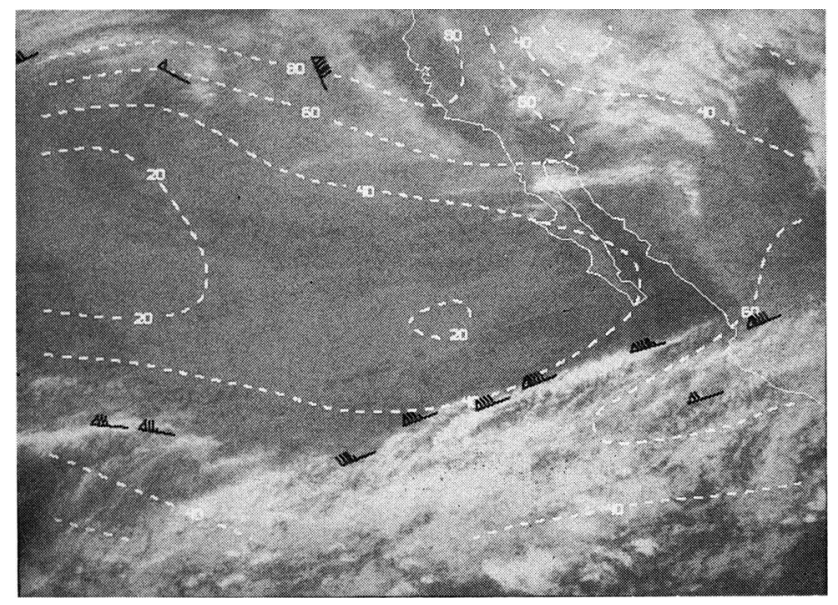

FIG. 4. $\mathrm{CO}_{2}$ ratio heights (black) compared with heights empirically estimated from the window channel brightness temperatures (white) from the upgraded winds algorithm for 1200 UTC 24 April 1990. 


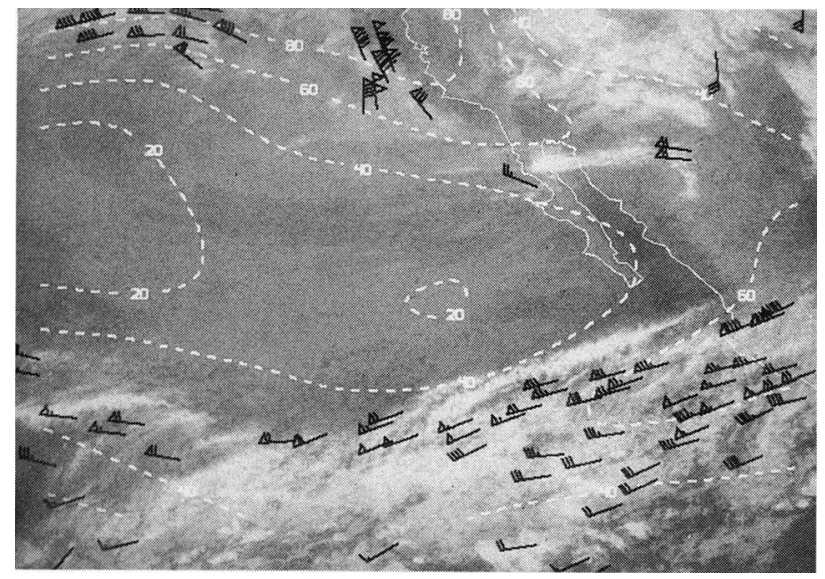

FIG. 5. Same as Fig. 3., but with demonstration winds $\left(\mathrm{CO}_{2}\right.$ heights and 15-min loops).

and gradient winds derived from VAS temperature retrievals were generated twice daily to supplement the operational winds in the vicinity of Atlantic basin tropical cyclones.

Based on the successes of the April 1990 demonstration and evaluation during the 1990 Atlantic hurricane season, operational implementation of the upgraded algorithm for full disk winds four times daily will come in 1991. At that time, low level winds will be produced using the picture-pair technique, and winds at all levels will be produced using an automated technique with $\mathrm{CO}_{2}$ heights and the combined set will beedited manually. Further model impact tests will be conducted by the NMC Development Division as well.
Acknowledgements. The experimental winds were produced by Chris Velden, Tim Schmit, Kurt Brueske, Tony Schreiner, and Robert Merrill of CIMSS, and Cecil Paris from Systems Design and Applications Branch, NESDIS. The operational winds were produced by the Synoptic Analysis Branch, NESDIS where Mark Ruminski deserves special mention for his extra efforts to inspect both operational and experimental wind products in real time. Curtis Holland of the Interactive Processing Branch, NESDIS provided valuable on site support at the VDUC. Dennis Deaven, Geoff DiMego, and Dennis Keyser of the Regional and Mesoscale Modelling Branch, Development Division, NMC assisted with the model impact tests. The VAS schedules were adjusted by the Satellite Operations Control Center, where Gordon Moiles was instrumental in satisfying the data needs for the demonstration. This work was supported by NOAA contract 50-WCNE-8-06058.

\section{References}

Eyre, J. R., and W. P. Menzel, 1989: Retrieval of cloud parameters from satellite sounder data: A simulation study. J. Appl. Meteor., 28, 267-275.

Green, R., G. Hughes, C. Novack and R. Schreitz, 1975: The automatic extraction of wind estimates from VISSR data. NOAA Technical Memorandum NESS 64, 94-110.

McIDAS Applications Guide, 1988: Cloud drift winds. A Space Science and Engineering Center Manual, Chap. 11.

Menzel, W. P., W. L. Smith and T. R. Stewart, 1983: Improved cloud motion wind vector and altitude assignment using VAS. J. Clim. Appl. Meteor., 22, 377-384.

Merrill, R., 1989: Advances in the automated production of wind estimates from geostationary satellite imaging. Fourth Conference on Satellite Meteorology, 16-19 May, San Diego, Amer. Meteor. Soc., 246-249. 